\title{
A Study on the Effective Long Type Coil Shape by Multidisciplinary Method in Induction Heating
}

\author{
Young Hwa Lee ${ }^{1}$, Yun Sok Ha ${ }^{2}$ \\ ${ }^{1}$ Department of Automation System, Dongyang Mirae University, Seoul, South Korea \\ ${ }^{2}$ Welding Research Part, Samsung Heavy Industries, Geoje, South Korea \\ Email: yhlee@dongyang.ac.kr
}

Received 31 January 2014; revised 4 March 2014; accepted 21 March 2014

Copyright (C) 2014 by authors and Scientific Research Publishing Inc.

This work is licensed under the Creative Commons Attribution International License (CC BY). http://creativecommons.org/licenses/by/4.0/

c) (i) Open Access

\section{Abstract}

The axis symmetric analysis method can neither handle initial curved plates nor be used in the optimization of coil shapes because an axis symmetric coil is the only shape to analyze in this method. But the method using some discrete divisions and steps, can overcome these difficulties and show more accurate, reasonable results of temperatures and deflections in flat or curved plates with initial curvature, than those in the axis symmetric analysis method. Traditionally, the coil shape in induction heating is circular shape and it needs the moving process along heating lines. To overcome these, the "long type coil" with some linear parallel coils is proposed. It does not need the moving process along heating lines and reduces the heating process time. The results of experiments are compared with those of simulation.

\section{Keywords}

Induction Heating, Electro-Magnetic Analysis, Heat Transfer Analysis, Inherent Strain

\section{Introduction}

Nowadays, the plate bending in shipbuilding yard uses a method of cold roller or a method of line heating by gas torch. But a hot induction heating is widely used as the method of plate bending in the field of civil architecture. To analyze the process of plate bending by induction heating, the multi-disciplinary analysis is required, including some methods such as an electro-magnetic analysis, a heat transfer analysis, and a deformation analysis. As one of this method, the axis-symmetry analysis method (ASAM) was proposed by Kim [1]. But this method could not model various shape of coil except with a circular shape and could not be used in the case that had 
different clearances between heating coils and plates with initial curvatures like concave, saddle, or twisted plates. It could be only used in a circular shape coil and constant clearance on a flat plate. Furthermore, it used only one time magnetic analysis, so could not consider the variance of parameters dependent on temperature. To overcome the restrictions of ASAM, the multi divisional analysis method (MDAM) was proposed by Lee [2]. It used some discrete divisions according to the moving direction and speed of coil, and was possible to handle the shape of coil and varying clearances. In this paper, the long type coil that is more efficient coil shape was proposed, made, and tested in the plate with initial curvature. After that, the results by test were compared with the results by MDAM.

\section{Multi Divisional Analysis Method}

\subsection{The Process of Analysis}

As being stated before, the analysis of plate bending by induction heating requires an electro-magnetic analysis, a heat transfer analysis composed of heating and cooling, and a deformation analysis. The process of the electromagnetic analysis and a heat transfer analysis is shown in Figure 1 and Figure 2.

\subsection{Analysis in a Circular Coil}

The first step of analysis in a circular coil is determining the division numbers to analyze according to the longitudinal direction in the shape of heating coil. Next step is modeling coil, core, and plate at each division. In Figure 3, the coil has a one turn circular shape with $10 \mathrm{~mm} \times 10 \mathrm{~mm}$ and $1 \mathrm{~mm}$ thickness as a cross section. So it has 5 divisions.

The concept drawing of analysis in a circular coil is shown in Figure 4. The coil is moving along the direction of heating line and the 5 divisions of different cross sections are passing through the dotted analysis line. Models of plate, air, and coil with 5 divisions in one turn circular coil on analysis line are shown in Figure 5. Each division is half model because of its bilateral symmetry.

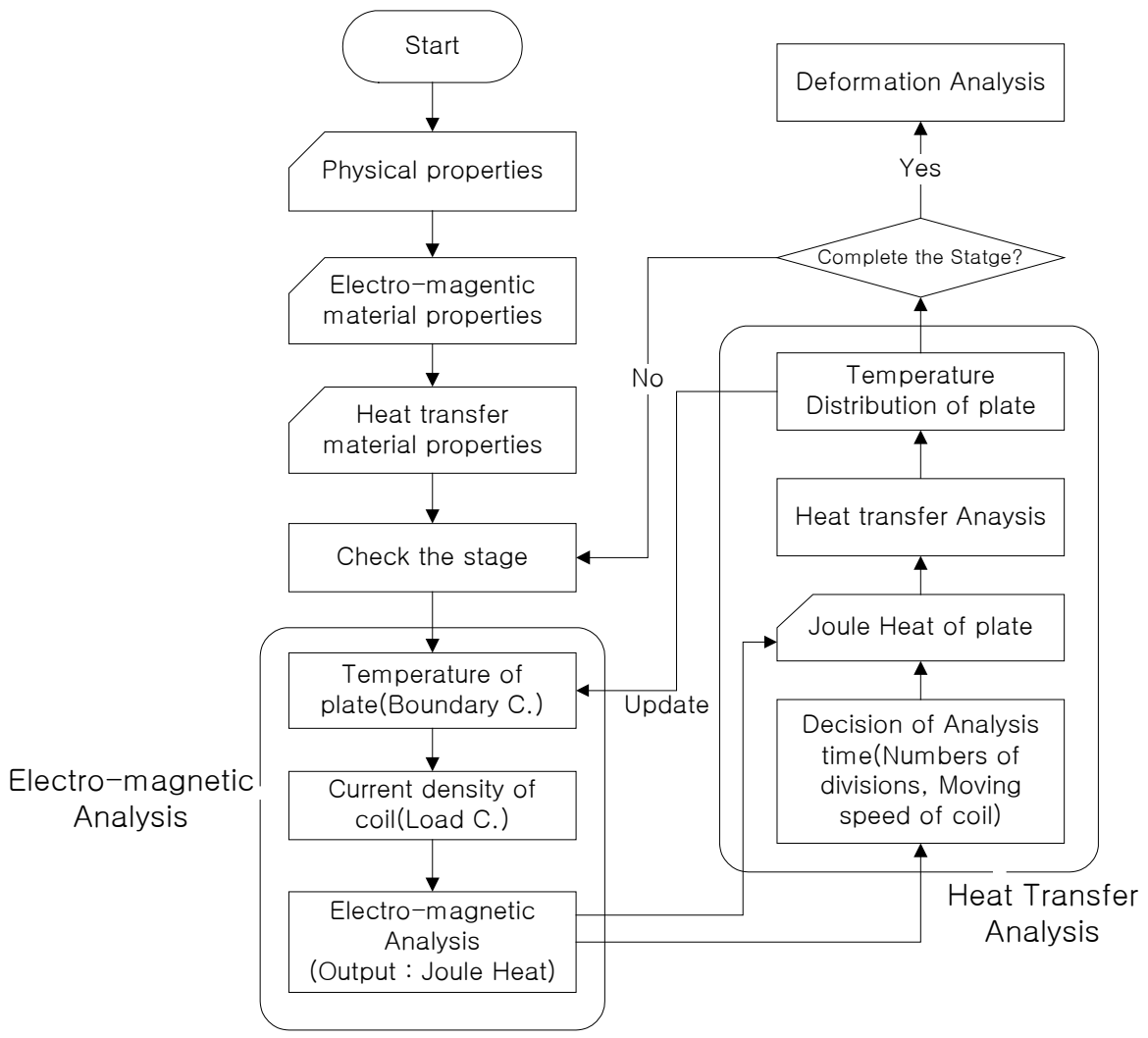

Figure 1. Flow chart of electro-magnetic and heat transfer analysis. 

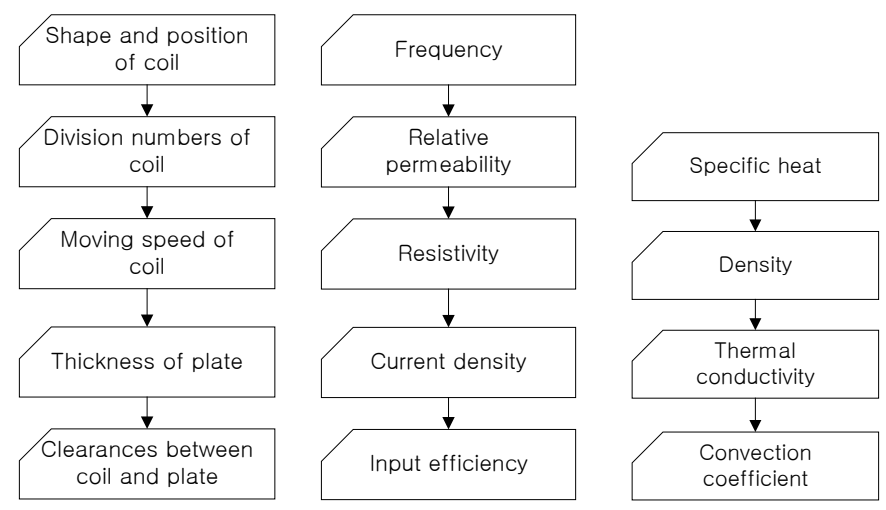

Figure 2. Local flow charts of physical properties, electro-magnetic material properties and heat transfer material properties.

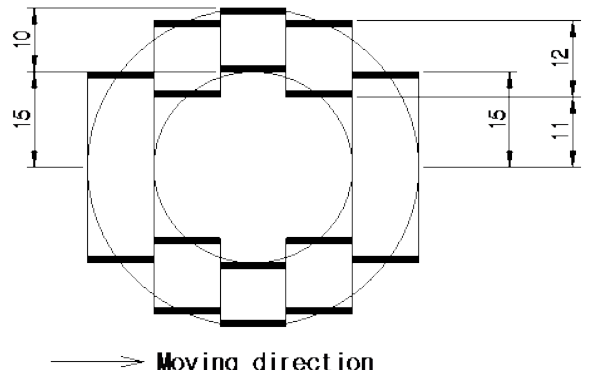

Model of 5 divisions in top view

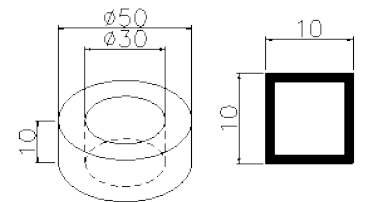

Isogonal view of coil and cross section of coil

Figure 3. Discrete divisions of coil in top view and isogonal view.

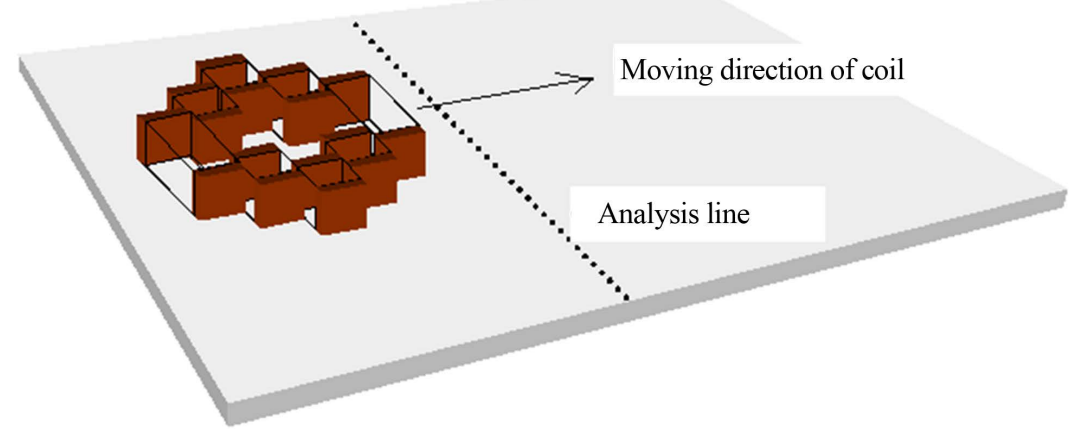

Figure 4. Three dimensional view of plate and coil with 5 divisions in one turn coil.
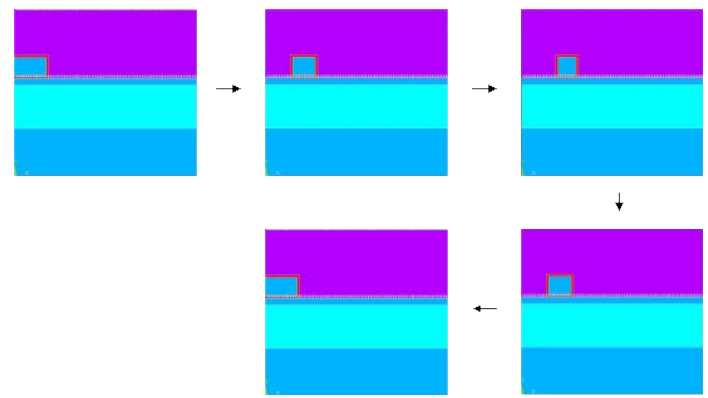

Figure 5. Models of plate, air, and coil with 5 divisions in one turn coil on analysis line (each division is half model because of its bilateral symmetry). 


\subsection{Analysis in a Long Type Coil}

In this paper a long type coil is proposed because it is more efficient for heating and it has more intensive concentration of heat than a circular coil. In a long type coil, the cross section is uniform along the longitudinal direction, so it does not require any division of coil. The proposed long type coil in heating system is shown in Figure 6.

Stronger advantage of a long type coil is that moving of coil is not necessary. If we know the optimal heating time, heating can be exerted during that time without moving, so the heating system can be simplified. On the other hand, moving of coil is necessary in the case of a conventional circular coil, so the heating system is more complex than the one of a long type coil. Resultantly, in the case of a long type coil, the heating efficiency is better, the equipment is simpler, the modeling of the cross section of coil for analysis is easier, and the modeling is more accurate because it has a constant cross section area.

After modeling, some data for electro-magnetic analysis and heat transfer analysis are necessary. The data for analysis are physical properties, electro-magnetic material properties, and heat transfer material properties. Physical properties are the shape and position of coil, division numbers of coil (these are not necessary in the case of a long type coil), moving speed of coil (this is not necessary in the case of a long type coil), thickness of plate, and clearances between coil and plate. Electro-magnetic material properties are frequency, relative permeability, resistivity, current density, and input efficiency. Heat transfer material properties are specific heat, density, thermal conductivity, and convection coefficient. Electro-magnetic analysis and heat transfer analysis using those data in a circular coil are repeated in each division stage but those in a long type coil are executed one time because a long type coil has one division stage. At the final stage of the analysis, the distribution of temperature of plate can be obtained and these are the input data for a deformation analysis based on inherent strain method.

\subsection{Comparison a Circular Coil with a Long Type Coil}

Coils that are being used in some shipbuilding yards have circular shape, and during heating the coils are moving according to heating lines which are same as the case of heating by a gas torch. But the proposed long type coil is possible for static heating onto the heating line. If the heating line is longer than the length of heating coil, some numbers of static heating can be applied.

At the viewpoint of modeling, the models of the first division and the last division of the circular coil in Figure 3 have modeling errors because the model of each division is the average cross section of each division. The thick line is the model of coil at each division in Figure 3 and error means the difference between thick line and curve of coil at each division. But in the long type coil in Figure 6, there is no error of modeling because it has constant cross section along the longitudinal direction of coil. More precise analysis is possible in the long type coil.

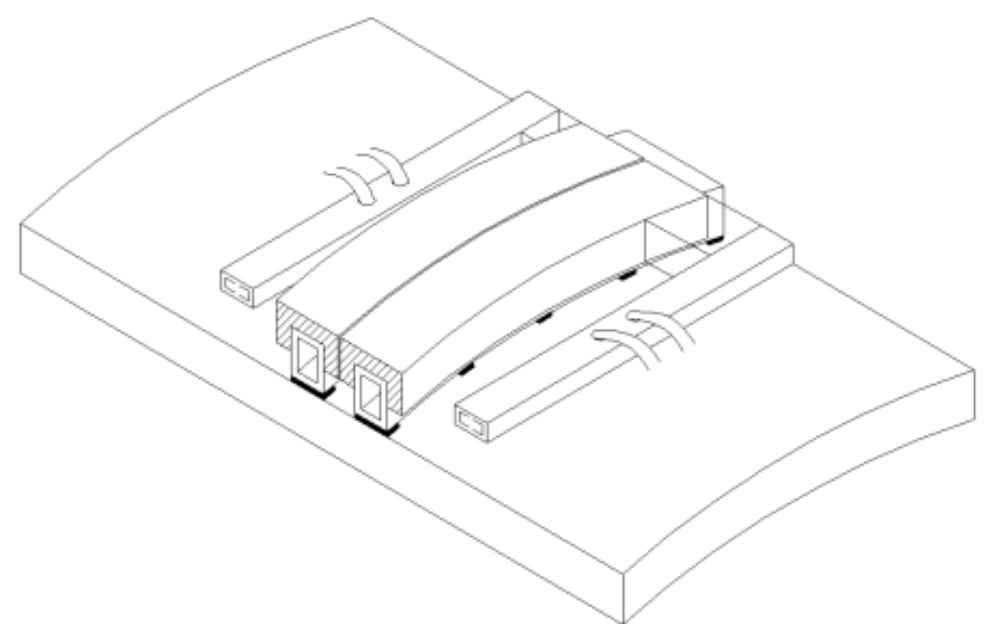

Figure 6. Configuration of a long type coil in the case of plate with initial curvature. 


\section{Heating Experiments and Analysis}

\subsection{The Process of Experiments}

The experiments to make second curvature of the plate with initial curvature were executed in the experimental models in Table 1.

Experimental models are curved plates that have the radii of 10,000 $\mathrm{mm}$ and $5000 \mathrm{~mm}$ and experiments are executed to make saddle plate with second curvature. The first curvature is made by roll press and the radius of mid part is more exact but the radii of both sides have smaller than that of mid part. The used power to heat is $160 \mathrm{~kW}$, voltage is $400 \mathrm{~V}$, and frequency is $4.5 \mathrm{~Hz}$ induced the optimal penetration depth [2].

Heating time was determined by the percentage of the region that was over the critical temperature each model [3]. Cooling is water cooling for rapid cooling and cooling time is the three times of heating time for enough cooling [2]. Heating time and cooling time are shown in Table 2 and the picture of heating experiment is shown in Figure 7. Elliptic heated region along the thickness direction can be found the edge of plate in Figure 7.

\subsection{Measurement of Deflection}

After experiments, deflections of plate were measured by 3-dimensional measuring machine in each model. The used measuring machine has the measuring range of length $1200 \mathrm{~mm}$, width $800 \mathrm{~mm}$, and height $700 \mathrm{~mm}$, and has the resolution of $0.5 \mu \mathrm{m}$ in three directions. Measuring lines and coordinate are shown in Figure 8 and the picture of measuring process is shown in Figure 9.

\subsection{Comparison Results of Experiments with Those of Analysis}

After experiments, the results of deflections were measured by 3-dimensional measuring machine in each model, and the deflections of simulation were obtained by the multi divisional analysis method [4]. Two results of the

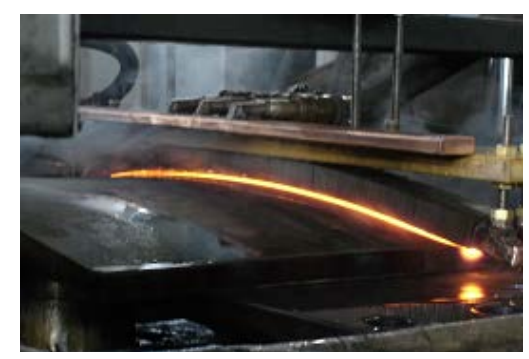

Figure 7. Picture of heating process.

Table 1. Experimental models.

\begin{tabular}{cccc}
\hline Model & Width $\times$ length $\times$ thickness $(\mathrm{mm})$ & Radius $(\mathrm{mm})$ & Material \\
\hline S12-10k & $400 \times 400 \times 12$ & 10,000 & Mild steel \\
S16-10k & $400 \times 400 \times 16$ & 10,000 & Mild steel \\
S12-5k & $400 \times 400 \times 12$ & 5000 & Mild steel \\
S16-5k & $400 \times 400 \times 16$ & 5000 & Mild steel \\
\hline
\end{tabular}

Table 2. Heating time and cooling time.

\begin{tabular}{ccc}
\hline Model & Heating time (sec) & Cooling time (sec) \\
S12-10k & 8 & 24 \\
S16-10k & 10 & 30 \\
S12-5k & 8 & 24 \\
S16-5k & 10 & 30 \\
\hline
\end{tabular}




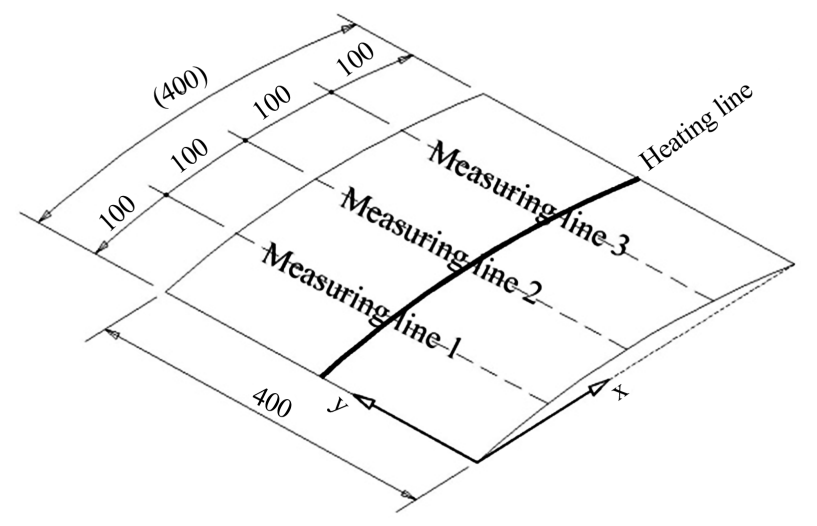

Figure 8. Measuring lines and coordinate.

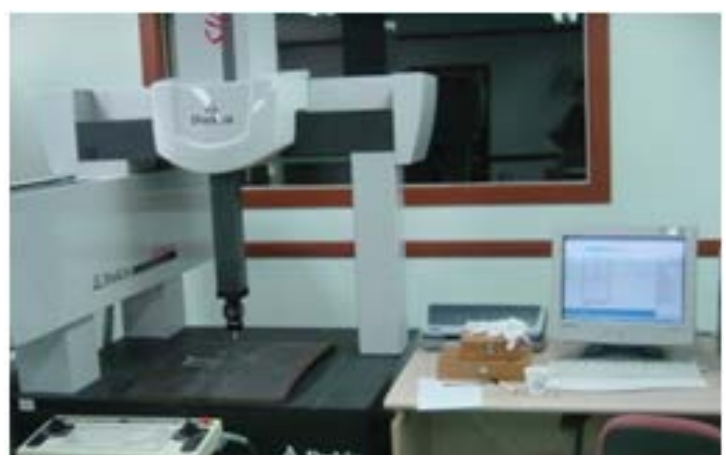

Figure 9. Picture of measuring process.

analysis and experiment are shown in Tables 3-6, and in Figures 10-13. In three tables, the deflections of three measuring lines that are located in $\mathrm{x}=100,200$, and $300 \mathrm{~mm}$. At the position $\mathrm{y}=200 \mathrm{~mm}$ that is midpoint, the deflection is maximum in each model. In three figures, each curve is the deflection by experiments and each node shape that is circle, rectangle, or asterisk is the deflection by analysis.

The results of deflections by analysis coincide generally with those by experiments. Maximum deflection accords each other, and curves of deflections by experiments are smooth, but node shapes of deflection by analysis have the "v" shape on the basis of mid part $(\mathrm{y}=200 \mathrm{~mm})$. It may be caused by the characteristic of the inherent strain method that is used to find the deflection of plate after the electro-magnetic analysis and heat transfer analysis. Inherent strain method [4] uses the inherent region can be found by searching the region that had reached over the critical temperature and at the edge boundary of it, the equivalent nodal forces can be calculated and elastic deformation analysis using these forces can calculate the deflection of a plate. Under the assumption that the region of phase transformation is the region of inherent strain, equivalent nodal force and moment of the heating line are calculated by inherent strain method and the maximum slope and deflection at mid part of the plate are calculated by the elastic deformation analysis [5]. The "Mx" is the nodal equivalent longitudinal moment in the direction of heating line, "my" in the nodal equivalent lateral moment per unit length along the heating line, " $\mathrm{Fx}$ " is the longitudinal force, and "fy" is the lateral force in Figure 14. The elastic deformation analysis using these two equivalent forces and two equivalent moments can find the deflections of plate, and these make the deflections of "V" shape.

\subsection{Comparison a Circular Coil with a Long Type Coil}

Comparison of a conventional circular coil with a proposed long type coil is shown in Table 7. It is under the condition that the final maximum deflection is same in the circular coil and long type coil. The result of the circular coil is the result of the experiment from Kim [1]. Plates are flat plates of mild steal that have the dimension of width $500 \mathrm{~mm}$, length $2000 \mathrm{~mm}$, and thickness $16 \mathrm{~mm}$. Heating condition is that one heating line in the position of mid-width caused the maximum deflection $1.05 \mathrm{~mm}$ at the midpoint. 
Table 3. Results of model S12-10k (unit: mm).

\begin{tabular}{ccccccc}
\hline \multirow{2}{*}{ Width y } & \multicolumn{2}{c}{ Line 1} & \multicolumn{2}{c}{ Line 2} & \multicolumn{2}{c}{ Line 3 } \\
\cline { 2 - 6 } & Analysis & Experiment & Analysis & Experiment & Analysis & Experiment \\
\hline 0 & 0.0000 & 0.0000 & 0.0000 & 0.0000 & 0.0000 & 0.0000 \\
40 & -0.2402 & -0.3256 & -0.2493 & -0.3128 & -0.2402 & -0.3359 \\
80 & -0.5756 & -0.6435 & -0.6003 & -0.6589 & -0.5756 & -0.6732 \\
120 & -0.8793 & -0.9897 & -0.9560 & -1.0048 & -0.8793 & -1.0026 \\
160 & -1.2666 & -1.3569 & -1.3146 & -1.3691 & -1.2666 & -1.3721 \\
200 & -1.6215 & -1.5687 & -1.6758 & -1.5790 & -1.6215 & -1.5590 \\
240 & -1.2666 & -1.3985 & -1.3146 & -1.4120 & -1.2666 & -1.4256 \\
280 & -0.8793 & -1.1598 & -0.9560 & -1.1751 & -0.8793 & -1.2009 \\
320 & -0.5756 & -0.7530 & -0.6003 & -0.8059 & -0.5756 & -0.8559 \\
360 & -0.2402 & -0.3987 & -0.2493 & -0.4159 & -0.2402 & -0.4398 \\
400 & 0.0000 & 0.0000 & 0.0000 & 0.0000 & 0.0000 & 0.0000 \\
\hline
\end{tabular}

Table 4. Results of model S16-10k (unit: mm).

\begin{tabular}{ccccccc}
\hline \multirow{2}{*}{ Width y } & \multicolumn{2}{c}{ Line 1} & \multicolumn{2}{c}{ Line 2} & \multicolumn{2}{c}{ Line 3} \\
\cline { 2 - 6 } & Analysis & Experiment & Analysis & Experiment & Analysis & Experiment \\
\hline 0 & 0.0000 & 0.0000 & 0.0000 & 0.0000 & 0.0000 & 0.0000 \\
40 & -0.1620 & -0.1912 & -0.1683 & -0.2356 & -0.1620 & -0.2324 \\
80 & -0.3916 & -0.4659 & -0.4083 & -0.4998 & -0.3916 & -0.5008 \\
120 & -0.6263 & -0.7598 & -0.6520 & -0.7751 & -0.6263 & -0.7895 \\
160 & -0.8656 & -0.9862 & -0.8978 & -0.9862 & -0.8656 & -0.9989 \\
200 & -1.1092 & -1.0894 & -1.1455 & -1.1059 & -1.1092 & -1.0833 \\
240 & -0.8656 & -0.9363 & -0.8978 & -0.9568 & -0.8656 & -0.9129 \\
280 & -0.6263 & -0.7298 & -0.6520 & -0.7598 & -0.6263 & -0.6968 \\
320 & -0.3916 & -0.4912 & -0.4083 & -0.5529 & -0.3916 & -0.4559 \\
\hline & -0.1620 & -0.2556 & -0.1683 & -0.2785 & -0.1620 & -0.2698 \\
\hline
\end{tabular}

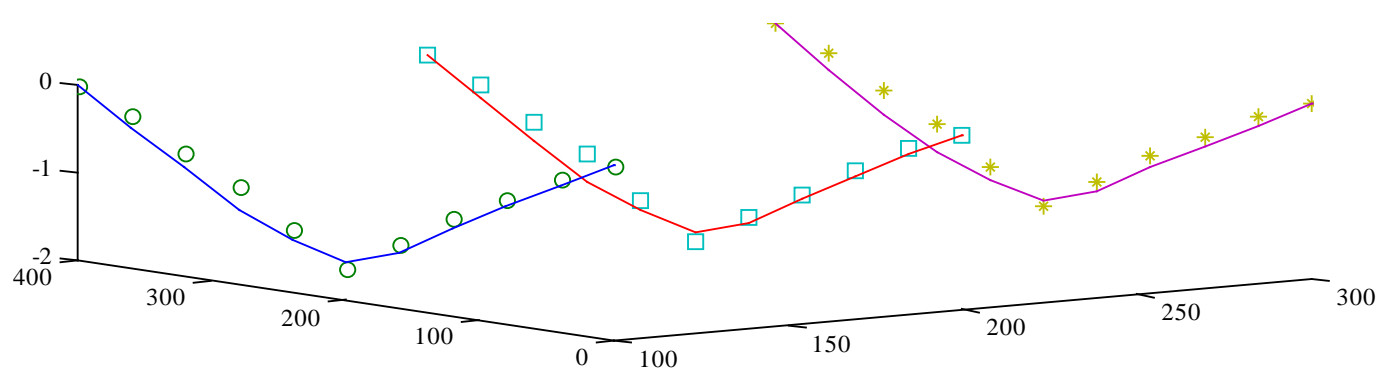

Figure 10. Deflection lines of model S12-10k. 
Table 5. Results of model S12-5k (unit: mm).

\begin{tabular}{ccccccc}
\hline \multirow{2}{*}{ Width y } & \multicolumn{2}{c}{ Line 1} & \multicolumn{2}{c}{ Line 2 } & \multicolumn{2}{c}{ Line 3 } \\
\cline { 2 - 6 } & Analysis & Experiment & Analysis & Experiment & Analysis & Experiment \\
\hline 0 & 0.0000 & 0.0000 & 0.0000 & 0.0000 & 0.0000 & 0.0000 \\
40 & -0.2368 & -0.3123 & -0.2466 & -0.3158 & -0.2368 & -0.3315 \\
80 & -0.5633 & -0.6709 & -0.5899 & -0.6987 & -0.5633 & -0.6965 \\
120 & -0.8930 & -0.9987 & -0.9346 & -1.0475 & -0.8930 & -1.0159 \\
160 & -1.2272 & -1.3125 & -1.2801 & -1.3654 & -1.2272 & -1.3356 \\
200 & -1.5665 & -1.5319 & -1.6273 & -1.5611 & -1.5665 & -1.5435 \\
240 & -1.2272 & -1.3482 & -1.2801 & -1.3789 & -1.2272 & -1.3712 \\
280 & -0.8930 & -1.0956 & -0.9346 & -1.0856 & -0.8930 & -1.1256 \\
320 & -0.5633 & -0.7187 & -0.5899 & -0.7233 & -0.5633 & -0.8001 \\
360 & -0.2368 & -0.3797 & -0.2466 & -0.4233 & -0.2368 & -0.4265 \\
400 & 0.0000 & 0.0000 & 0.0000 & 0.0000 & 0.0000 & 0.0000 \\
\hline
\end{tabular}

Table 6. Results of model S16-5k (unit: mm).

\begin{tabular}{|c|c|c|c|c|c|c|}
\hline \multirow{2}{*}{ Width y } & \multicolumn{2}{|c|}{ Line 1} & \multicolumn{2}{|c|}{ Line 2} & \multicolumn{2}{|c|}{ Line 3} \\
\hline & Analysis & Experiment & Analysis & Experiment & Analysis & Experiment \\
\hline 0 & 0.0000 & 0.0000 & 0.0000 & 0.0000 & 0.0000 & 0.0000 \\
\hline 40 & -0.1607 & -0.1802 & -0.1671 & -0.2311 & -0.1607 & -0.1978 \\
\hline 80 & -0.3867 & -0.4123 & -0.4042 & -0.4453 & -0.3867 & -0.4256 \\
\hline 120 & -0.6164 & -0.6751 & -0.6434 & -0.6999 & -0.6164 & -0.6853 \\
\hline 160 & -0.8499 & -0.8753 & -0.8841 & -0.8865 & -0.8499 & -0.8859 \\
\hline 200 & -1.0872 & -1.0089 & -1.1262 & -1.0236 & -1.0872 & -0.9896 \\
\hline 240 & -0.8499 & -0.8875 & -0.8841 & -0.8957 & -0.8499 & -0.8995 \\
\hline 280 & -0.6164 & -0.6892 & -0.6434 & -0.7129 & -0.6164 & -0.7314 \\
\hline 320 & -0.3867 & -0.4365 & -0.4042 & -0.4869 & -0.3867 & -0.4912 \\
\hline 360 & -0.1607 & -0.2356 & -0.1671 & -0.2569 & -0.1607 & -0.2571 \\
\hline 400 & 0.0000 & 0.0000 & 0.0000 & 0.0000 & 0.0000 & 0.0000 \\
\hline
\end{tabular}

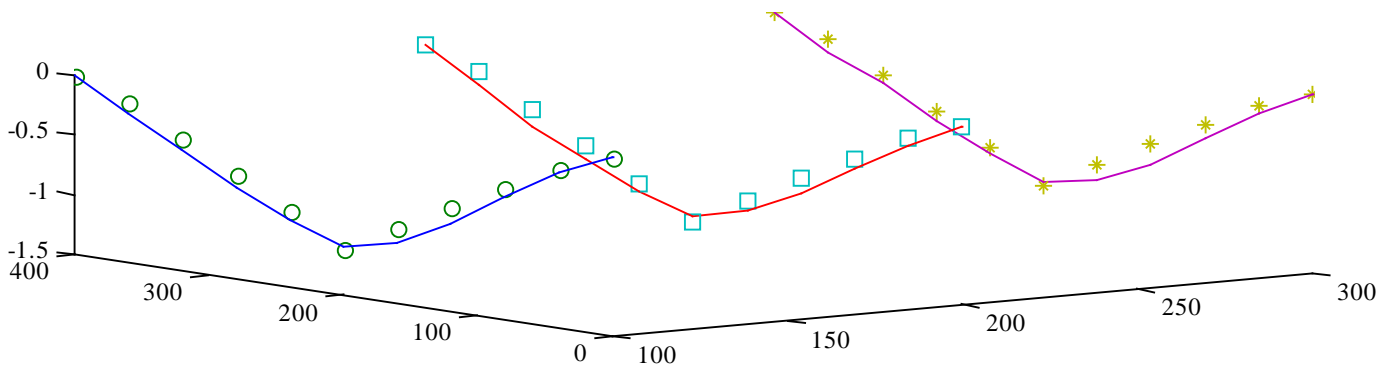

Figure 11. Deflection lines of model S16-10k. 


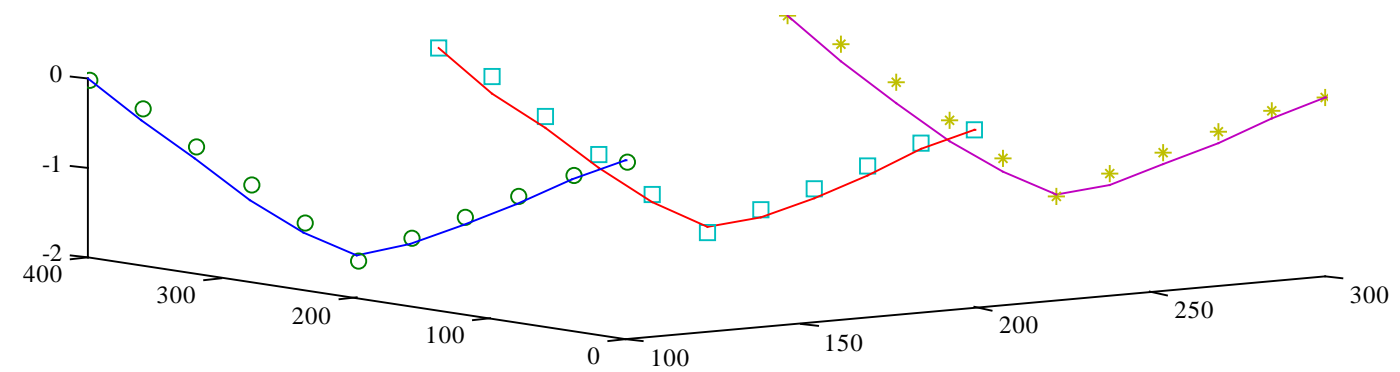

Figure 12. Deflection lines of model S12-5k.

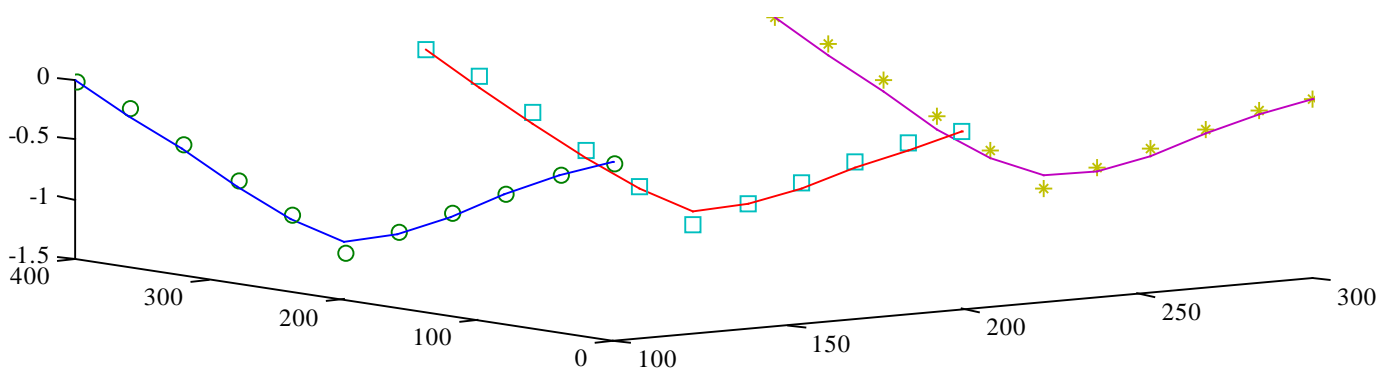

Figure 13. Deflection lines of model S16-5k.

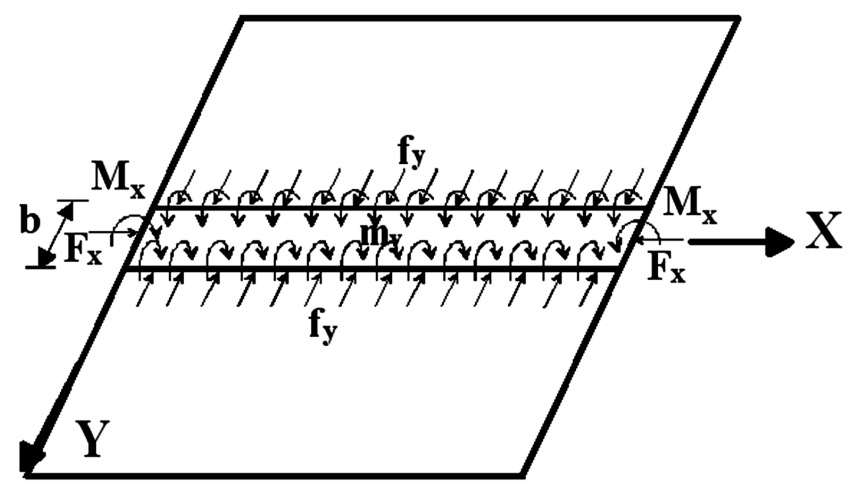

Figure 14. Equivalent nodal force and moment along heating line.

Table 7. Comparison of the energy efficiency in the circular coil and long type coil.

\begin{tabular}{ccc}
\hline Item & Circular coil & Long type coil \\
\hline Moving speed & $\mathrm{V}=17 \mathrm{~mm} / \mathrm{sec}$ & - \\
Length of heating line & $2 \mathrm{~m}$ & $2 \mathrm{~m}$ \\
Heating time & $118 \mathrm{sec}$ & $10 \mathrm{sec}$ \\
Heating time efficiency & 1 (criterion) & 11.8 \\
Feature & Moving heating & Static heating \\
Power & $80 \mathrm{~kW}$ & $450 \mathrm{~kW}$ \\
Energy & $9440 \mathrm{~kJ} / 2.62 \mathrm{kWh}$ & $4500 \mathrm{~kJ} / 1.25 \mathrm{kWh}$ \\
Energy efficiency & 1 (criterion) & 2.1 \\
\hline
\end{tabular}

According to the comparison, the heating time efficiency of long type coil is better 11.8 times than the circular coil because long type coil was used as static heating. But the necessary power of long type coil is bigger 
than the circular coil. The used energy can be obtained by multiplying power by heating time. Long type coil has the shorter heating time and bigger power. The energy of long type coil is $1.25 \mathrm{kWh}$ and the circular coil is 2.62 $\mathrm{kWh}$. At last, long type coil is faster 11.8 times in heating time, and better 2.1 times in energy efficiency.

\section{Conclusions}

Conventional axis-symmetry analysis method (ASAM) could be only used in a circular shape coil and the constant clearance in a flat plate because it used the axis-symmetry model. Furthermore it used only one time magnetic analysis, so it could not consider the variance of parameters dependent on temperature.

In the improved simulation program of induction heating, the multi divisional analysis method (MDAM) was proposed by Lee [2] to overcome the restriction of shape of coil, clearance between coil and plate, and consideration of the variance of parameters dependent on temperature. It used some discrete divisions according to the moving direction and speed of coil, and did repetitive electro-magnetic analysis and heat transfer analysis at each division, so it could model any shape of coils and plates with initial curvatures, and it also considered the variance of parameters dependent on temperature.

Using the multi divisional analysis method, a better shape of coil was tried to find out, and a long type coil was proposed in this paper. It can be used without moving the coil, but a conventional circular coil is used with moving the coil on the other hand. Long type coil was made, and experimented on the plate that had initial curvature. The comparison of the deflection by the proposed multi divisional analysis method with the deflection of the experiment had good coincidence.

According to the comparison of the proposed long type coil with the conventional circular coil under the condition that the final maximum deflection is same, the heating time efficiency of long type coil is better 11.8 times than the circular coil because long type coil can be used as static heating. Additionally long type coil is better 2.1 times in energy efficiency.

\section{Acknowledgements}

This works was supported by Dongyang Mirae University under the Academic Research Program.

\section{References}

[1] Kim, H.K. (2002) Simulation of Line Heating by High Frequency Induction Heating. Ph.D. Thesis, Seoul National University, Seoul.

[2] Lee, Y.H. (2006) Development of the Induction Heating Machine and Analysis Program for a Plate Bending. Ph.D. Thesis, Seoul National University, Seoul.

[3] Marder, A.R. and Krauss, G. (1970) The Effect of Morphology on the Strength of Lath Martensite. Proceedings of the 2nd International Conference on the Strength of Met and Alloys, 3, American Society for Metals, Metals Park, 822 823.

[4] Lee, Y.H. (2012) Prediction of Plate Bending by Multi Divisional Analysis in Induction Heating. Journal of Ship Research, 56, 146-153. http://dx.doi.org/10.5957/JOSR.56.3.110007

[5] Jang, C.D., Seo, S.I. and Ko, D.E. (1997) A Study on the Prediction of Deformations of Plates Due to Line Heating Using a Simplified Thermal Elasto-Plastic Analysis. Journal of Ship Production, 13, 22-27. 